\title{
Erratum to: Macronutrient (N, P, K) and Redoximorphic Metal (Fe, Mn) Allocation in Leersia oryzoides (Rice Cutgrass) Grown Under Different Flood Regimes
}

\author{
Samuel C. Pierce • Matt T. Moore • Dan Larsen •
}

S. R. Pezeshki

Published online: 1 September 2012

(C) Springer Science+Business Media B.V. 2012

\section{Erratum to: Water Air Soil Pollut DOI 10.1007/s11270-009-0120-y}

The original publication of this article (doi:10.1007/ s11270-009-0120-y) unfortunately contained errors.

Table 2. Values for K, Fe, and Mn are all an order of magnitude too high. The values have been decreased by a factor of ten, and rounded to under four significant figures.

Figure 2. Aboveground Fe concentrations are actually two orders of magnitude higher than shown in the original figure. For the sake of simplicity, all units have been changed to $\mathrm{mg} / \mathrm{L}$ and the height of the bars reduced in the aboveground treatment to reflect this change. The lowercase letters elucidating treatment

The online version of the original article can be found at http:// dx.doi.org/10.1007/s11270-009-0120-y.

S. C. Pierce $(\bowtie) \cdot$ S. R. Pezeshki

Department of Biology, The University of Memphis,

Memphis, TN 38152, USA

e-mail: spierce1@memphis.edu

S. C. Pierce

e-mail: spierce@cfr.msstate.edu

M. T. Moore

USDA-Agricultural Research Service,

National Sedimentation Laboratory,

P.O. Box 1157, Oxford, MS 38655, USA differences in shoot tissue were not included in the updated graph, as there was no treatment effect.

Figure 3. Mn concentrations are actually two orders of magnitude higher than shown. The units have been changed to $\mathrm{mg} / \mathrm{L}$ and the scale on the $\mathrm{Y}$-axis has been decreased by an order of magnitude.

The following text should be removed: Discussion, page 81 , column 2 , paragraph 2 , sentence 3 (running from lines 22-25 of right column). "Root Fe concentrations in Chen et al., (2005) were, however, more than an order of magnitude higher than those in the present study."

The corrected Table 2, Fig. 2, and Fig. 3 are reproduced in the next page.

D. Larsen

Department of Earth Sciences, The University of Memphis, Memphis, TN 38152, USA

Present Address:

S. C. Pierce

Department of Wildlife, Fisheries, and Aquaculture,

Mississippi State University,

Mississippi State, MS 39762, USA 
Table 2 Mean biomass and total nutrient uptake across treatments

\begin{tabular}{ccccc}
\hline & Control & $\begin{array}{l}\text { Intermittently } \\
\text { flooded }\end{array}$ & $\begin{array}{l}\text { Partially } \\
\text { flooded }\end{array}$ & $\begin{array}{l}\text { Continuously } \\
\text { flooded }\end{array}$ \\
\hline Biomass (g) & & & & \\
Belowground & $23.3 \pm 6.6$ & $22.2 \pm 6.4$ & $23 \pm 9.2$ & $19.8 \pm 5.6$ \\
Aboveground & $38.3 \pm 12$ & $38.8 \pm 9.1$ & $37.3 \pm 8.9$ & $45.7 \pm 12$ \\
N (mg) & & & & \\
Belowground & $124.0 \pm 68$ & $120.4 \pm 66$ & $109.1 \pm 54$ & $98.6 \pm 46$ \\
Aboveground & $277.7 \pm 88$ & $225 \pm 99$ & $264 \pm 100$ & $303 \pm 134$ \\
P (mg) & & & & \\
Belowground & $35.3 \pm 15$ & $49.4 \pm 29$ & $57.0 \pm 29$ & $53.0 \pm 16$ \\
Aboveground & $82.1 \pm 20$ & $67.7 \pm 24$ & $70.2 \pm 24$ & $94.8 \pm 38$ \\
K (mg) & & & & \\
Belowground & $143 \pm 39$ & $138 \pm 61$ & $103 \pm 30$ & $99 \pm 45$ \\
Aboveground & $356 \pm 129$ & $347 \pm 108$ & $263 \pm 84$ & $349 \pm 91$ \\
Fe (mg) & & & & \\
Belowground & $47.3 \pm 20$ & $68.9 \pm 43$ & $113 \pm 74$ & $130 \pm 45^{\mathrm{a}}$ \\
Aboveground & $5.95 \pm 2.8$ & $8.29 \pm 4.1$ & $8.11 \pm 8.1$ & $8.21 \pm 3.7$ \\
Mn (mg) & & & & \\
Belowground & $2.67 \pm 08$ & $4.53 \pm 2.0$ & $5.88 \pm 3.8$ & $5.71 \pm 2.4$ \\
Aboveground & $3.16 \pm 1.4$ & $8.07 \pm 2.7$ & $10.7 \pm 4.2^{\mathrm{a}}$ & $13.5 \pm 5.8^{\mathrm{a}}$ \\
\hline
\end{tabular}

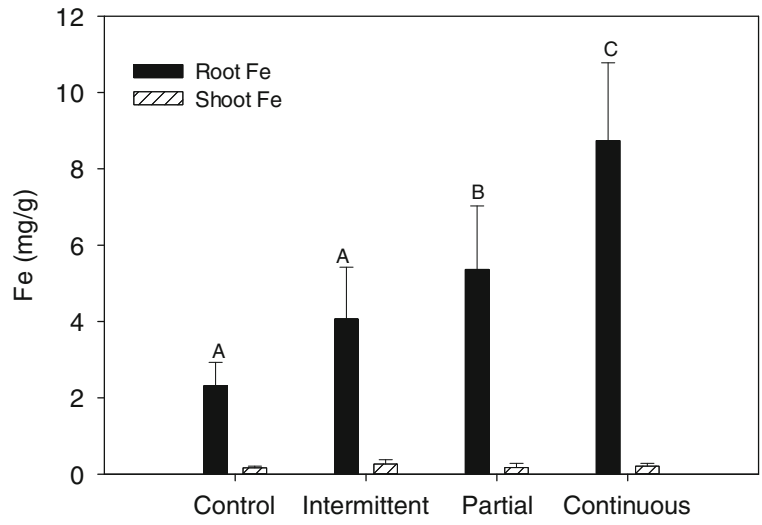

Fig. 2 Fe tissue concentrations

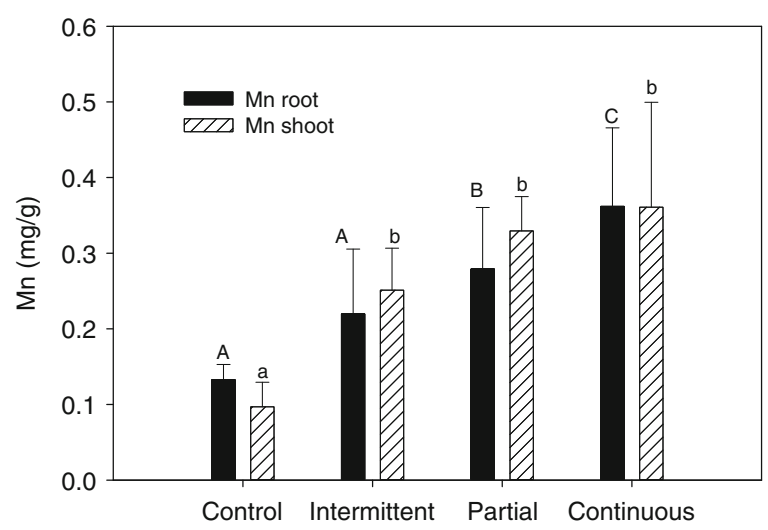

Fig. 3 Mn tissue concentrations 